\title{
Alignment of Business, Architecture, Process, and Organisation in a Software Development Context
}

\author{
Stefanie Betz \\ Blekinge Institute of Technology \\ 37179 Karlskrona, Sweden \\ +46-(0)455-385852 \\ stefanie.betz@bth.se
}

\author{
Claes Wohlin \\ Blekinge Institute of Technology \\ 37179 Karlskrona, Sweden \\ +46-(0)455-385820 \\ claes.wohlin@bth.se
}

\begin{abstract}
In this paper we investigate the current state of work regarding alignment of Business, Architecture, Process, and Organisation (BAPO) perspectives in a software product development context. We planned to do that by conducting a systematic literature study to capture the state of the art in alignment of BAPO in software development. But, as it turned out we found that almost no substantial information is available about the alignment of BAPO in software development. Thus, based on the available literature and a small qualitative study, we defined a conceptual model of the alignment of BAPO including five levels of alignment that can be used as a basis for future empirical studies.
\end{abstract}

\section{Categories and Subject Descriptors}

D.2.9 [Software Engineering]: Management - Life cycle, Software process models, Software quality assurance.

\section{General Terms}

Management, Measurement, Documentation, Economics.

\section{Keywords}

BAPO, Business, Architecture, Process, Organisation, Alignment, Decision, Software Engineering.

\section{INTRODUCTION}

In [1] it is stated how the ideal process in terms of alignment of BAPO in software product development should look like and that the BAPO perspectives are dependent on each other. Meaning if one perspective is altered the other perspectives will be affected.

Thus, we assume that dependencies between the BAPO perspectives exist and as such the different perspectives affect each other. But, they are not necessarily aligned to the level where the other perspectives are fully taken into account before taking a decision with respect to one of the perspectives. Representatives of other perspectives are not involved in the decision-making process so that a proactive alignment between the perspectives can take place e.g. architectural design needs to be taken into account when going global [2]. So, the question is what the consequences are if the alignment is missing and what the actual level of alignment present in a company is and what measures are taken to align them, or if it is assumed that they will eventually align as they are affected. In order to study that we developed a

\footnotetext{
Permission to make digital or hard copies of all or part of this work for personal or classroom use is granted without fee provided that copies are not made or distributed for profit or commercial advantage and that copies bear this notice and the full citation on the first page. To copy otherwise, or republish, to post on servers or to redistribute to lists, requires prior specific permission and/or a fee.

ESEM'12, September 19-20, 2012, Lund, Sweden.

Copyright 2012 ACM 978-1-4503-1056-7/12/09...\$15.00
}

conceptual model of the alignment of BAPO with five levels of alignment based on an aborted Systematic Literature Study (SLR) and a small interview study.

In our SLR we found that almost no substantial information is available about the alignment of BAPO at least not in (global) software product development. But, it is stated that it is important to understand the dependencies between the BAPO perspectives [2]. Therefore, it is important to define the different BAPO perspectives and their level of alignment. Thus, we provide a conceptual model of BAPO including five levels of alignment to provide a basis for a common understanding of the BAPO perspectives and their possible level of alignment.

The remainder of the paper is structured as follows. The next section describes the aborted SLR. In Section 3, we present our interview study. Section 4 contains a detailed description of the developed conceptual model including the five levels of alignment and the paper ends with a summary and future work.

\section{SYSTEMATIC LITERATURE REVIEW}

The goal of our SLR was to find out if there has been "any work" done regarding the alignment of BAPO in a global software product development context. Any work can be: empirical studies, models, frameworks or theories [3]. Thus, our research question was:

RQ: What is the state-of-the-art regarding the alignment of the four perspectives business, architecture, process, and organisation in a global software product development context?

We added the global software development (GSD) context because we thought in a distributed setting the alignment is even more important for example architectural design needs to be taken into account when going global [2]. Furthermore, adding GSD is a possibility to narrow the scope in the literature study because business, architecture, process and organisation are words which are used quite often and in a lot of different contexts (e.g. development processes, business processes, process improvement, process maturity and so on).

\subsection{Search strategy}

To answer the research question a systematic review was conducted. As a first step we looked for search strings. We aimed at finding the state of the art regarding alignment in a global software development product context. Thus, we included the four perspectives business, architecture, process, and organisation in our search strings. In addition, we were looking for alignment and its synonyms and we used GSD and its synonyms as well as embedded systems and its synonyms for further refinement of the search in our pilot study. But, the plan to use Google scholar did not work the way we intended, because Google scholar is not able 
to handle the search string with several ANDs and ORs. This means only one group of words can be chosen as "one of the given synonyms should match". Therefore we got very different results, because we either needed to add the group with an AND to the search string by choosing only one of the synonyms (e.g. AND "Alignment") or we had to put the whole group in the section "only one of the words" (e.g. OR "Alignment", "Traceability", "Goals", "Integration", "Bridge", "Harmony", "Linkage") but then we could not add any other groups to the OR section because there is only one OR section for all. Due to that problem with the search string the results were very different given the number of hits and the general output. In general, it could be shown the outcome had a strong variation in terms of hits as well as in terms of research directions. As a cross check we tested the original search string using the database SciVerse Scopus, but the result was also not useful, we only got 28 hits and the papers have not been relevant for our search, as we judged based on the titles. Thus, we decided to reduce the search string. In doing so, we finally found one search string that at least included one of the known key papers [1] in the first 10 hits. Thus, we decided to use this search string to create a set of papers to be used for snowballing, i.e. given that we did not really find a good way of identifying the most relevant papers through searching databases. In addition, snowballing is found to be effective in identifying relevant research [4]. So, the final search string was:

"Business", "Architecture", "Process", "Organisation", "Alignment", and "Embedded Systems" in the AND section and "Global Software Development" OR "Global Software Engineering" OR "Distributed Software Development" OR "Distributed Development" OR "Offshoring" OR "Outsourcing" OR "Software Transfer" (number of hits 426).

In addition, the search strategy was based on the following parameters [5]:

Spatial parameters: global due to the actual setting

Temporal parameters: As we do snowballing, we cannot use the only most recent papers, due to references search on that paper. We will search backwards through references and we have no time limits there as well as in the initial search. In addition, we are looking on any work done in our specific topic.

Disciplinary parameters: We are aware that we are conducting an interdisciplinary search and expect that the software product line, the requirements engineering and the information system communities have done some work regarding the alignment of BAPO. But, we want to find out what work is available on our specific topic. Later, we are planning to compare existing approaches from the mentioned disciplines

Formal parameters: We are taking into account journals, conference and workshop proceedings (=peer reviewed).

Search applied on: Full text. We did choose the full text in order to get access to all the papers that do not include our keywords in title or abstract, although they may be relevant.

Language: English

Searched databases: Google scholar

Our search strategy was to take the 20 best hits from Google scholar as a start for snowballing.

\subsection{Assessment criteria and quality criteria}

As a second step before conducting the search and the literature selection we invented relevance assessment and quality criteria in order to be able to conduct a relevance analysis upon full text and conduct inclusion or exclusion [6]. Our relevance assessment criteria are shown in Table 1. In order to measure the relevance we used the following scoring: 1 point for yes (main questions), 0 point for unclear (main questions and sub questions), 0 point for no (main questions and sub questions), $1 / 4$ point for one of the areas of BAPO (sub questions), $1 / 2$ point for aligning two areas (sub questions), and $3 / 4$ point for aligning 3 areas (sub questions). If the full paper had more than 3 points, then it was considered as relevant and included.

Table 1. Relevance assessment criteria

\begin{tabular}{|l|}
\hline 1. Are the areas to align (BAPO) discussed in the paper? \\
\hline If not which of the following areas are included? \\
\hline a) Business; b) Architecture; c) Process and d) Organisation \\
\hline $\begin{array}{l}\text { 2. Is the paper dealing with (any kind of) alignment of all four areas } \\
\text { (BAPO)? }\end{array}$ \\
\hline If not, is the paper dealing with the alignment of some of the areas? \\
\hline Two at the time: a) B - A; b) B - P; c) B - O; d) A - P; e) A - O; f) P - O; \\
\hline Three at the time: g) B - A - P; h) A - P - O; i) B - P - O; j) B - A - O; \\
\hline 3. Is the setting a global software development project? \\
\hline 4. Is the regarded product embedded? \\
\hline 5. Is the paper concerned with software development \\
\hline
\end{tabular}

For the quality assessment we used the criteria in Table 2.

Table 2. Quality assessment criteria

\begin{tabular}{l}
\hline 1. Is the research aim clearly stated? \\
\hline 2. Is the research methodology clearly described? \\
\hline 3. Is the method chosen appropriate (e.g. answers the RQ)? \\
\hline $\begin{array}{l}\text { 4. Is the setting the study/method/theory applies to thoroughly and clearly } \\
\text { described }\end{array}$ \\
\hline 5. Are limitations and or validity threats discussed? \\
\hline 6. Does the empirical data and or results support the conclusion? \\
In order to measure the quality we used the following scoring: $1 / 2$ \\
point yes, 1/4 point partially, and 0 point for no. We did not define \\
a threshold, because the quality assessment was not used for \\
selecting papers. An external researcher with experience in \\
performing SLRs checked our assessment criteria and the scoring. \\
In addition we piloted the protocol.
\end{tabular}

\subsection{Literature selection}

In this phase, the aim was to choose the papers to be used as the basis for the actual snowballing. The literature selection was performed in three steps (see Table 3).

Table 3. Literature selection based on [6]

\begin{tabular}{l|l|l|l} 
Analysis Steps & $\begin{array}{l}\text { Inclusion } \\
\text { Criteria }\end{array}$ & $\begin{array}{l}\text { Researchers } \\
\text { involved }\end{array}$ & $\begin{array}{l}\text { Papers } \\
\text { left }\end{array}$ \\
\hline $\begin{array}{l}\text { 1. Selection based } \\
\text { on search }\end{array}$ & $\begin{array}{l}\text { Search strategy } \\
\text { parameters }\end{array}$ & $\begin{array}{l}\text { Leading } \\
\text { researcher }\end{array}$ & 20 \\
\hline $\begin{array}{l}\text { 2. Exclusion based } \\
\text { on title and abstract }\end{array}$ & Two researchers & 10 \\
\hline $\begin{array}{l}\text { 3. Exclusion based } \\
\text { on full paper }\end{array}$ & $\begin{array}{l}\text { Relevance } \\
\text { assessment }\end{array}$ & Two researchers & 0
\end{tabular}

Using the search string defined in Section 2.1, we got 426 hits. From these hits we took the first 20 hits fitting our search strategy parameters (see step 1). In the next step, step 2, we conducted the exclusion based on title and abstract. In order to do so both researchers separately assessed the titles and abstracts of the 20 
papers. This was followed by a discussion between the two researchers to obtain consensus on inclusion and exclusion. The researchers agreed on 14 out of 20 papers and decided for the 6 papers not in agreement to include all papers with at least one "inclusion" for the full text analysis. In step 3, we conducted a relevance analysis of the full text based on the relevance assessment criteria. Therefore, three papers have been randomly chosen as an input, none of the papers passed the relevance threshold. Then the "key paper" [1] has been chosen for relevance assessment but even there we did not find the paper relevant for the objectives of our SLR. Thus, being not able to find any relevant papers with our search strategy, we decided to check how the outcome of the search would be if we search without the GSD focus (search string: "Business", "Architecture", "Process", "Organisation", "Alignment", and "Embedded Systems"). We got more then 10.000 hits and in the list of the first 30 hits we found couple of identical papers to the ones found with the search string including the GSD focus. But, in general there was a stronger focus on software product lines and on business. Nevertheless, the papers either have already been assessed or not relevant for our search, as we judged based on the titles. At that stage we decided to abort the SLR because we could not find any relevant papers.

So, we found evidence that there is a lack of research regarding the alignment of the four perspectives business, architecture, process, and organisation in a software product development context. But, we found hints in the literature that dependencies between the different perspectives exist [1] and that alignment is needed between different perspectives [2]. Consequently, we conducted an interview study with a software product developing company to find out if there is a need to conduct research in this topic.

\section{INTERVIEW STUDY}

\subsection{Design of study}

To understand the level of alignment and challenges related to any misalignment, an interview study was conducted at a large company developing software-intensive systems for an international market. The interviews were planned to be semistructured in the sense that several areas should be covered in the interviews, but it was also important to allow the interviewees to express their opinions and experience in particular in relation to their specific expertise. In total, 11 people coming from a mixture of roles were interviewed. The roles include system architects, product managers, line managers on different levels and technical experts. The starting point for the interviews was the assumption that alignment of the different perspectives in BAPO is beneficial. Furthermore, it is assumed that it is preferable if the order of the letters in BAPO reflects the starting point for alignment, which is also argued in [1], although some iteration is most likely needed.

\subsection{Main findings}

Bellow, the findings in relation to the BAPO perspectives are summarized:

Business: There is an agreement that the business is changing quickly, although it is expressed slightly different by different interviewees. This puts requirements on the other letters in BAPO, i.e. APO.

Architecture: The development processes have changed over the last five years from supporting large plan-driven project to becoming more agile and lean. However, system and software architecture has not changed sufficiently with the processes. This also includes that the architecture is not necessarily well suited for distributed development. At the same time there is too little focus on the actual architecture (lack of long-term investments), and hence the development is too much driven by the need to deliver new features. Development becomes reactive and not proactive.

Process: To respond to the requirements from the market in terms of being faster to the market and being able to react to change quickly, agile and lean methods have been adopted. However, the consequence is that agile and lean software development is introduced without necessarily the business becoming agile as a whole or for that matter the system being ready for being developed using agile and lean software development.

Organisation: This perspective was mentioned the least by the interviewees than the other perspectives. There is a perception that the organisation indirectly decides too much, i.e. the "O" in BAPO becomes too powerful. The organisation was originally designed to fit the system architecture, but now it is the other way around, i.e. the organisation governs the architecture.

From the interviews, we conclude that the perspectives are not taken into account as much as needed and that alignment between the BAPO perspectives would most likely be beneficial. All perspectives have challenges regarding alignment with the other perspectives, e.g. architecture does not fit to a business decision, processes are agile but the business is not, and organisation governs architecture. Thus, to mitigate misalignment and to be able to make informed decisions there is a need to achieve a certain level of alignment. Based on the insights gained by literature and the conducted interview study, we decided to build a conceptual model of BAPO alignment including different levels of alignment. The aim of the model is it to use it as a basis for future empirical studies on the alignment of BAPO in a software product development context.

\section{CONCEPTUAL MODEL OF BAPO}

First, we are defining the four different perspectives business, architecture, process, and organisation. We tried to define the perspectives based on existing work as well as keeping the definitions short but precise in order to use them in an industrial context. As such the first two definitions are based on the definitions of [1], the third one is a shorter version of the definition of business processes from [7] and the fourth one is based on [8] and [9].

Business: how to make money from products and services,

Architecture: the structure to build the software,

Process: a set of structured activities to achieve a specific goal,

Organisation: a set of people organized in a specific structure to achieve a common goal.

Our conceptual model consists of the four BAPO perspectives, their dependencies, their attributes, and the levels of alignment. Figure 1 gives an overview over the BAPO perspectives and their dependencies. The attributes and levels of alignment are further elaborated below.

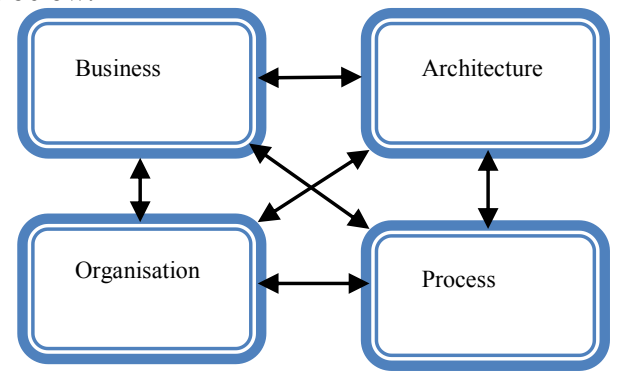

Figure 1. The BAPO perspectives 
The links between the different perspectives show the dependencies between the different perspectives. In our view the different perspectives are all dependent on each other, but often they are not well aligned as shown through the interview study. This implies that when a decision has been taken in one of the perspectives, for example, taking a business decision to develop a software system globally instead of at one site may have implications for the other perspectives. With a lack of alignment and a limited awareness of the dependencies the decisions may be made without taking the other perspectives into account. As a consequence a reactive process starts now, where the other perspectives will slowly align with potential (negative) effects on cost, quality and time as illustrated by the architecture perspective in the interview study in Section 3.2. Thus, we assume dependencies exist and alignment will eventually be achieved through being affected but a conscious alignment is preferable to be proactive towards changes caused by the dependencies. In our model each of the four perspectives has the following attributes to capture the decisions associated with them:

- Drivers: What are the drivers of the decisions?

- Responsible: Who is responsible for the decisions?

- Constraints: What are the constraints of the decisions?

- Objectives: What are the objectives of the decisions?

- Timeline: What is the timeline for the implementation of the decisions?

- Challenges: What are the major challenges of the implementation of decisions?

- Dependencies: What are the dependencies of the decisions?

Additionally, we developed five levels of alignment going from reactive to proactive decision-making.

1. Not aware or aware and ignored: none of the other perspectives is taken into account,

2. Aware: other perspectives are taken into account by the decision-maker, but no further actions are taken,

3. Informed: other perspectives are taken into account by the decision-maker and other stakeholders are informed that changes are needed after the decision is taken,

4. Communication: other perspectives are taken into account by the decision-maker and others are informed before the decision is taken that changes are needed,

5. Discussion: other perspectives are taken into account by the decision-maker and representatives of the other perspectives are involved in the decision-making process.

The levels are classified in reactive levels and proactive levels.

- Levels 1 and 2: reactive

- Level 3: from reactive to proactive - neutral

- Levels 4 and 5: proactive

The goal is to use the levels to classify the current situation of alignment of the BAPO perspectives in a company. Therefore, we also classified them according to their reactive and proactive level. Level 3 is on the borderline where the level is actually changing from reactive to proactive decision alignment.

\section{SUMMARY AND FUTURE WORK}

In this paper we described an aborted SLR on what research has been done regarding the alignment of the four perspectives business, architecture, process, and organisation in a (global) software product development context as well as a small interview study on the topic. As we found a research gap regarding the alignment of BAPO in software product development we presented a conceptual model of the alignment of the four BAPO perspectives including five levels of alignment of BAPO. With the help of the conceptual model we want to investigate decisions in relation to BAPO to understand if decisions made within one of the BAPO perspectives are taking the other perspectives into account. Thus, in our future work we want to investigate the actual state of the conscious alignment of the BAPO perspectives, or if the alignment is a slow process based on being affected. The objective is to capture this empirically. Moreover, we want to give companies the possibility to take the consequences of decisionmaking on the different perspective of BAPO into account as early as possible in the decision-making process. The goal is that the company is able to increase the level of alignment and as such evolve from taking reactive decisions to proactive decisions.

\section{ACKNOWLEDGMENTS}

This work is part of the BESQ+ research project funded by the Knowledge Foundation (grant: 20100311) in Sweden.

\section{REFERENCES}

[1] Linden, F. v. d., Bosch, J., Kamsties, E., Känsälä, K. and Obbink, H. 2004. Software Product Family Evaluation. In Software Product Line Conference (SPLC), 110-129.

[2] Mustapic, G., Wall, A., Norström, C., Crnkovic, I., Sandström, K.; Fröberg, J. and Andersson, J. 2004. Real world influences on software architecture - interviews with industrial system experts. In 4th Working Conference on Software Architecture (WICSA'04), 2004, 101-112.

[3] Webster, J. and Watson, R. T. 2002. Analyzing the past to prepare for the future: Writing a literature review. MIS Quarterly, vol. 26, no. 2, 13-23.

[4] Greenhalgh, T. and Peacock, K. R. 2005. Effectiveness and efficiency of search methods in systematic reviews of complex evidence: audit of primary sources. BMJ - Information in Practice, no. 331, 1064-1065.

[5] Duff, A. 1996. The literature search: a library-based model for information skills instruction. Library Review, 14-18.

[6] Kitchenham, B. and Charters, S. 2007. Guidelines for Performing Systematic Literature Reviews in Software Engineering. Software Engineering Group, School of Computer Science and Mathematics, Keele University, EBSE Technical Report Version 2.3.

[7] Davenport, T. H. and Short, J. E. 1990. The New Industrial Engineering: Information Technology and Business Process Reedesign. Sloan Management Review, Vol. 31, no. 4, 11-27.

[8] Daft, R.L. 2003. Organization Theory and Design, 8th ed., South-Western College Pub.

[9] Oxford University Press. 2012. Oxford Dictionaries. http://oxforddictionaries.com/definition/organization 